GT2008-50499

\title{
A SEMI-ANALYTICAL METHOD FOR THE SOLUTION OF ENTRANCE FLOW EFFECTS IN INHERENTLY RESTRICTED AEROSTATIC BEARINGS
}

\author{
Tobias Waumans* \\ Farid Al-Bender \\ Dominiek Reynaerts \\ Department of Mechanical Engineering \\ Katholieke Universiteit Leuven \\ Celestijnelaan 300B, 3001 Leuven \\ Belgium \\ Email: tobias.waumans@mech.kuleuven.be
}

\section{ABSTRACT}

The behaviour of aerostatic bearings is strongly influenced by the entrance flow effects nearby feeding sources. A basic understanding of these flow phenomena and accurate prediction of their relevant parameters are therefore essential in the design and optimisation process of any aerostatic bearing application. The subject matter of this paper has for long been the topic of extensive research. An overview of the different approaches found in literature shows mostly methods based on empirical formulas with a validity limited to the experimental conditions that produced them.

The proposed solution method uses the technique of separation of variables to convert the boundary-layer equations describing the laminar channel flow into an initial value problem. This allows the exact calculation of the pressure profile from gap entrance up to the attainment of viscous flow. Knowledge of the pressure distribution near the gap entrance together with the mass flow rate leads to the determination of an expedient coeffcient of discharge, allowing a more practical lumped parameter formulation.

The results are compared with experimental data from various sources and the agreement is found to be remarkably good, indicating that a laminar flow model is adequate in characterising the entrance flow over a wide range of working parameters.

*Address all correspondence to this author.

$\begin{array}{ll}\text { NOMENCLATURE } \\ m(I) & \text { characteristic value function } \\ \dot{m} & \text { mass flow rate } \\ \dot{m}_{o} & \text { entrance flow rate } \\ \dot{m}_{\text {feed }} & \text { feed flow rate } \\ \dot{m}_{\text {film }} & \text { film flow rate } \\ n(I) & \text { characteristic value function } \\ h & \text { gap height } \\ h_{a} & \text { exit gap height } \\ h_{o} & \text { entrance gap height } \\ p & \text { absolute pressure } \\ p_{a} & \text { ambient pressure } \\ p_{r} & \text { recess pressure } \\ p_{s} & \text { supply pressure } \\ p_{o} & \text { pressure at entrance gap } \\ p_{t} & \text { theoretical inlet pressure } \\ r & \text { radial distance from bearing centre } \\ r_{a} & \text { bearing outer radius } \\ r_{o} & \text { entrance gap radius } \\ \bar{u} & \text { main flow average velocity } \\ u, v, w & \text { velocity components } \\ x, y, z & \text { space coordinates } \\ A_{o} & \text { entrance curtain surface } \\ B_{v} & \text { pressure distribution function } \\ C_{d} & \text { coefficient of discharge } \\ C_{d, \text { orifice }} & \text { orifice coefficient of discharge } \\ & \\ & \end{array}$




\begin{tabular}{ll}
$G$ & velocity profile function \\
$H$ & normalised gap height \\
$I$ & velocity profile integral function \\
$M_{o}$ & entrance Mach number \\
$P$ & normalised pressure \\
$R$ & normalised radial distance \\
$R e_{o}$ & Reynolds number based on channel width \\
$R e_{o}^{*}$ & reduced Reynolds number \\
$T_{S}$ & fluid temperature at stagnation \\
$Q$ & velocity amplitude function \\
$U, V, W$ & normalised velocity components \\
$X, Y, Z$ & normalised space coordinates \\
Greek symbols & \\
$\gamma$ & polytropic exponent \\
$\kappa$ & ratio of specific heats of a gas \\
$\mu$ & dynamic viscosity \\
$\nu$ & normalised conicity angle \\
$\rho$ & density \\
$\Lambda_{e}$ & entrance number \\
$\Phi$ & nozzle function \\
$\wp$ & normalised density $=\rho / \rho_{o}$ \\
$\Re$ & universal gas constant divided by \\
& molecular weight \\
Subscripts & \\
$a$ & ambient value \\
$o$ & gap/channel entrance value \\
$S$ & stagnation value \\
$t$ & theoretical value \\
& \\
\hline &
\end{tabular}

\section{INTRODUCTION}

Interest in the problem of entrance flow in aerostatic bearings dates back to many decades ago. A basic understanding of the effects and a correct prediction of the relevant parameters has proven to be essential in the successful application of aerostatic bearings. As no general formula exists to quantify the entrance pressure (or mass flow), a range of mostly empirical methods has been developed to allow the solution of the complete flow problem. A comprehensive overview of these methods will be given later on. The value of the results produced by empirical methods is however always limited to the underlying experimental conditions that produced them.

In a wider context, the stated problem of entrance flow nearby feeding sources of fluid film bearings can be regarded as an example of a narrow-channel internal flow configuration. This broad class of flow phenomena is of both practical and theoretical importance in fluid mechanics: flow intake devices, plane ducts, radial diffusers etc. The solution method proposed in this paper is easily adaptable to describe the flow effects encountered in these applications.

In order to situate the present work, a brief overview of the air bearing research activities at the department of Mechani- cal Engineering of the KULeuven is presented as a background. The research started in the early seventies and concentrated initially on the development of design methods to improve aerostatic bearing characteristics as load-carrying capacity, stiffness and air consumption. Later on, bearing designs with a convergent gap geometry were explored as they yield far better properties when compared to conventional uniform gap geometries. More recent work focuses on air bearings with an active controlled conformable gap allowing the realisation of virtually infinite stiff bearings. Besides this, a considerable amount of research oriented towards the development of a design methodology to prevent phenomena as pneumatic instabilities (hammering). A good reference concerning this is given in [1].

The first section of this paper outlines the problem configuration and its notation; thereafter, a brief overview of other solution methods for the stated problem is provided. Further on, the method of separation of variables is applied to the laminar boundary-layer equations describing narrow-channel flow, resulting in a begin value problem. This set of equations is then solved for different bearing configurations and feeding systems. Comparison of the results with experimentally obtained data shows a good agreement. Finally, the formulation of an expedient coefficient of discharge leads to a lumped parameter formulation of the problem, suitable for practical bearing design.

\section{BEARING CONFIGURATION AND PROBLEM NOTA- TION}

Some typical aerostatic bearing configurations are shown in Fig. 1. Nearby each feeding source, entrance flow effects occur before the flow settles down to a viscous regime. A detail view of this entrance region and corresponding pressure distribution for (diverging) radial flow are shown in Fig. 2. When starting from the supply side of the bearing, three different flow regions are distinguished:

(1) The feed flow region, in which the flow quickly accelerates from stagnation up to gap entrance $\left(p_{s} \rightarrow p_{o}\right)$;

(2) The (channel) entrance flow region, in which both inertial and viscous forces are of equal importance, is characterised by a typical trough and subsequent crest in the pressure curve $\left(p_{o} \rightarrow \ldots\right)$;

(3) The fully developed flow region where viscous forces prevail. This region constitutes the greatest portion of a normal bearing and extents up to gap exit where ambient conditions are present $\left(\ldots \rightarrow p_{a}\right)$.

The theoretical inlet pressure $p_{t}$ is defined by extrapolating the pressure curve of the viscous flow region back to gap entrance. This pressure value is needed to determine the entrance head loss (both a gain/recovery $\left(p_{t}>p_{o}\right)$ and head loss $\left(p_{t}<p_{o}\right)$ are observed in radial diverging flow). Assuming the flow to be 


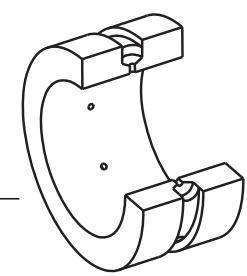

(a) journal bearing
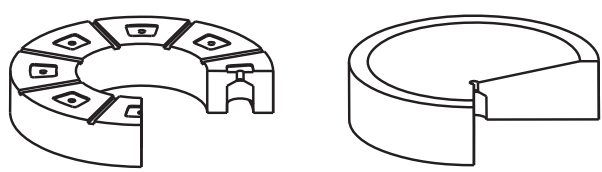

(NOT TO SCALE)

(b) thrust bearing

(c) convergent pad
Figure 1. TYPICAL AEROSTATIC BEARING CONFIGURATIONS.

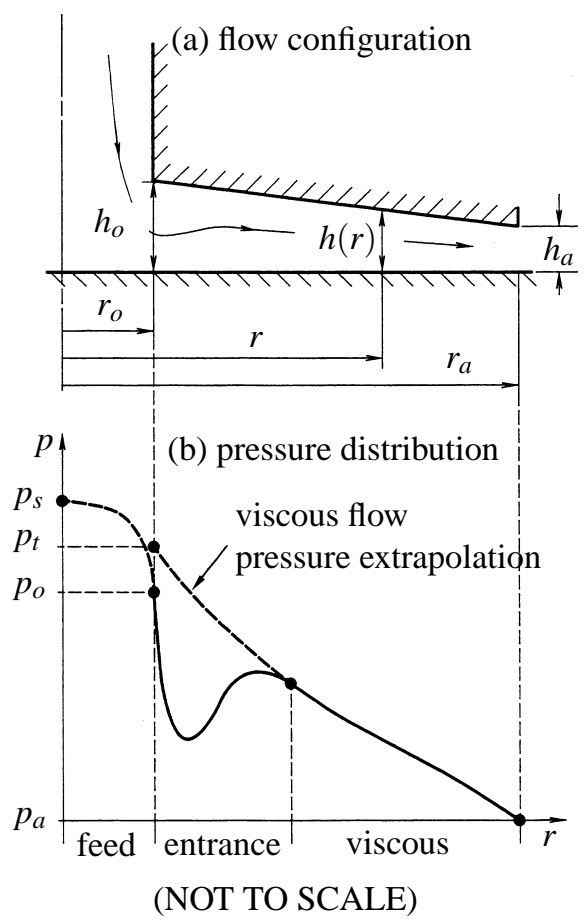

Figure 2. ENTRANCE REGION AND NOTATION.

fully viscous over the entire bearing area, results only in a negligible error on the load carrying capacity. However, accurate determination of this theoretical inlet pressure $p_{t}$ is still required as a necessary and sufficient condition in solving the viscous flow problem.

\section{SHORT OVERVIEW OF OTHER METHODS}

Many attempts have been made to understand and quantify the entrance effects encountered nearby feeding sources of aerostatic bearings. Only a brief overview of these attempts will be given in this paper. A much more extensive discussion regarding this topic can be found in [2].

A first class of mostly empirical methods simplifies the prob- lem to a lumped parameter formulation which relates the mass flow rate $\dot{m}$ (or equivalent $p_{o}$ ) to $p_{s}$ and $p_{t}$. The main problem lies in determining the discharge or loss coefficient of these formulations. The orifice/nozzle formula and Vohr's correlation formula [3] are the most widely known examples of this first class. Although a lot of experimental work has been carried out to quantify the entrance loss coefficient of both methods, satisfactory empirical formulas seem either complicated or have only a very limited validity. Another conclusion concerning these two methods is that the experimentally obtained data is only assumed to be function of the Reynolds number and some other geometrical parameters. The large scatter on the data points that have been related in this way, indicates that also the entrance Mach number must be taken into account to obtain a good correlation. A last comment concerning lumped parameter models points to the fact that although practical to the bearing designer, no information about the actual flow development downstream of the feeding source is gained.

Other solution methods are based on the approximation of the inertia term, momentum integral principles or series expansions. The results are in all cases approximative and for some methods hard to implement.

A last category holds the different numerical solutions applied to the entrance problem. When utilising numerical schemes, care must be taken to ensure convergence of the solution process. This fact together with the large amount of computing time, make these methods rather unhandy for practical bearing design. However, recent studies by Helene et al. [4], Braun and Dzodzo [5], Renn and Hsiao [6] indicate their value for purpose of comparison and for handling turbulent flow conditions or non-axisymmetric geometries.

\section{METHOD OF SEPARATION OF VARIABLES From stagnation up to gap entrance}

As stated above, the entire problem from stagnation to gap exit consists of three different flow regimes. First, the flow accelerates from stagnation (supply plenum $p_{s}$ ) up to gap entrance $\left(p_{o}\right)$. It can be shown that the flow in this region is purely inertial or inviscid. Further assumption of adiabatic expansion and neglecting the time dependent terms, leads to the quasi-steady Euler equation. As the actual pressure distribution in this region is of no interest in this work, a lumped parameter formulation relating conditions at supply side to gap entrance, is sufficient:

$$
\dot{m}_{o}=2 \pi r_{o} h_{o} \sqrt{\frac{2 \kappa}{\kappa-1}} \frac{p_{s}}{\sqrt{\Re T_{S}}} \Phi\left(\bar{p}_{o}\right)
$$

where, 


$$
\Phi\left(\bar{p}_{o}\right)=\left\{\begin{array}{lll}
\sqrt{\bar{p}_{o}^{2 / \kappa}-\bar{p}_{o}^{(\kappa+1) / \kappa}} & \text { for } & \bar{p}_{o} \geq \bar{p}_{c} \\
\sqrt{\bar{p}_{c}^{2 / \kappa}-\bar{p}_{c}^{(\kappa+1) / \kappa}} & \text { for } & \bar{p}_{o} \leq \bar{p}_{c}
\end{array}\right.
$$

with,

$$
\begin{aligned}
& \bar{p}_{o}=\frac{p_{o}}{p_{s}} \\
& \bar{p}_{c}=\left(\frac{2}{\kappa+1}\right)^{\kappa /(\kappa-1)}
\end{aligned}
$$

\section{Entrance flow}

At gap entrance the flow transforms from a uniform velocity distribution towards viscous flow with a parabolic distribution. In this second region, flow can be described by Prandtl's boundarylayer equations which are derived as a first order approximation of the Navier-Stokes equations. This simplification is justified by the fact that the stated problem possesses two length scales, being the transversal scale (film thickness $h$ ) and the in-plane scale (radial distance $r$ ), and $h \ll r$. Further downstream and up to gap exit, flow will settle down to fully developed viscous flow with a parabolic velocity profile. In this third flow region, inertia terms have only a limited effect. Omitting these inertia terms leads to a second order approximation which is only applicable in this last viscous region. This approximation forms the essence of the well known Reynolds lubrication theory.

The proposed solution method focuses on this second flow region described by the boundary-layer equations. The underlying theory of the method is given in [7]. It is worth mentioning that the basic idea of the solution method was stated by Tang [8]. He started from an already truncated form of the equation of motion and had to introduce further approximation to arrive to a solution. The below set of equations has been deduced in a completely analytical way and is therefore an exact solution of the idea of Tang. For the case of axisymmetric radial compressible flow, separating the velocity into an amplitude function $Q(R)$ and profile function $G(R, Z)$ :

$$
U(R, Z)=Q(R) G(R, Z)
$$

with velocity integral function,

$$
I=\int_{0}^{1} G d Z
$$

allows the initial problem to be reformulated as:

$$
\left\{\begin{array}{l}
Q^{\prime}=\frac{1}{H^{2} R e_{o}^{*} \wp} m(I) \\
P^{\prime}=\frac{-\kappa M_{o}^{2} Q}{H^{2} e_{o}^{*}} n(I) \\
I=\frac{1}{R H \wp Q}
\end{array}\right.
$$

where the primes denote the first derivative with respect to $R$. Figure 3 clarifies the configuration and its notation. The height function $H$, radial distance $R$ and density function $\wp$ are normalised with respect to the upstream gap entrance values:

$$
\begin{aligned}
H & =h / h_{o} \\
R & =r / r_{o} \\
\wp & =\rho / \rho_{o}
\end{aligned}
$$

The reduced Reynolds number $R e_{o}^{*}$ and entrance Mach number $M_{o}$ are defined as:

$$
\begin{aligned}
R e_{o} & =\frac{\rho_{o} \bar{u}_{o} r_{o}}{\mu_{o}} \\
R e_{o}^{*} & =R e_{o}\left(\frac{h_{o}}{r_{o}}\right)^{2} \\
M_{o} & =\frac{\bar{u}_{o}}{\sqrt{\kappa p_{o} / \rho_{o}}}
\end{aligned}
$$

The velocity integral function $I$ ranges from 1 to 0 , with $I=1$ corresponding to a uniform velocity profile, $I=2 / 3$ to a parabolic profile and $I \leq 0.457$ indicating the onset of reverse flow. The characteristic value functions $m(I)$ and $n(I)$ are tabulated over the full range of $I$ with steps of $\Delta I=0.01$. Intermediate values are obtained by cubic interpolation. Reference [7] explains in more detail how these functions are derived.

The problem has now been converted to an initial value problem, which one can solve readily for a given geometry, fluid, Reynolds number $R e_{o}^{*}$ and entrance Mach number $M_{o}$. In our case a Runge-Kutta method of the 4 th order was applied. The following discussion elaborates on the different boundary conditions, flow conditions and solution procedure of the method.

\section{Boundary condition on $I$}

At gap entrance, the flow is assumed to have a uniform velocity profile $\left(I_{o}=1\right)$. This can be justified by the following reasoning. For small values of $h_{o} / r_{o}$, the converging accelerating 


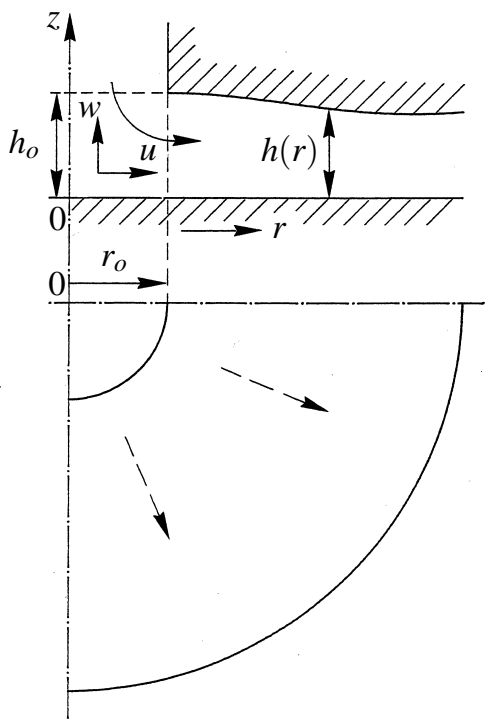

Figure 3. RADIAL FLOW NOTATION

flow from stagnation to gap entrance resembles a plane sink flow just upstream the entrance curtain. This means that the boundarylayer thickness is gradually decreasing as the flow approaches the gap entrance. Just before entering the gap, the thickness of the boundary-layer is proportional to the inverse of the square root of the Reynolds number Re based on the film thickness (Ref. [9], p. 153). Typical values of $R e$ are in the order of $10^{3}$, implying a boundary-layer thickness to gap height ratio of less than 0.05 which corresponds to a value $I_{o}$ greater than 0.9. Taking into account that velocity profiles values near unity are very similar, only little error will be introduced by this assumption.

For diverging flow, the integrated velocity profile function always undershoots its parabolic value and approaches it asymptotically from below (Fig. 4). At high values of the reduced Reynolds number $R e_{o}^{*}$, this undershoot becomes more pronounced. From a certain value on, $I$ drops below 0.457 and backflow occurs. Although back-flow does not directly implicate the onset of permanent turbulence, since the flow may reattach further downstream. Nevertheless, some basic assumptions of the solution method are undermined if large regions of back-flow are present.

\section{Density function $\wp$ in the gap}

The polytropic equation of state for an ideal gas is given by:

$$
\wp=P^{1 / \gamma}
$$

in which for simplicity the isothermal expansion will be supposed $(\gamma=1)$. Although this assumption leads to no significant

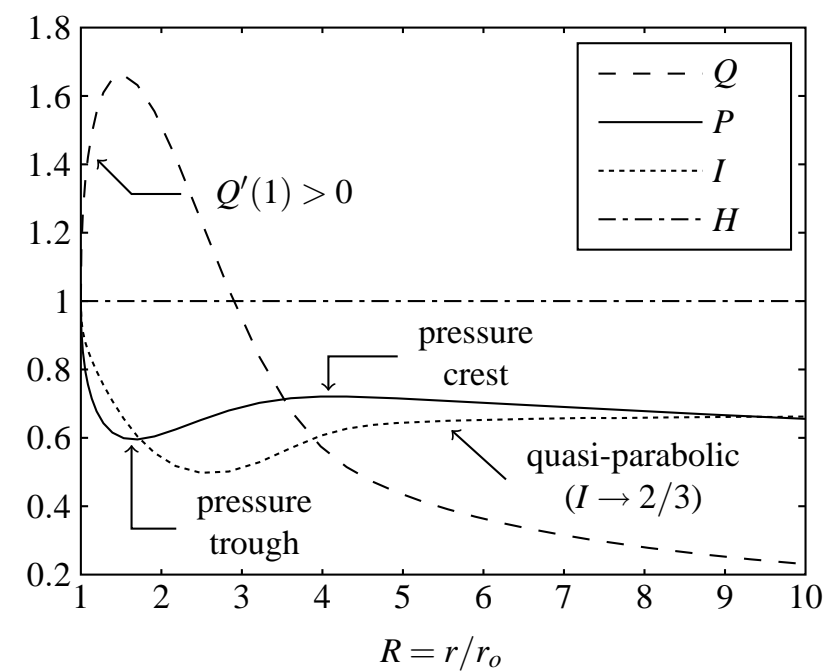

Figure 4. TYPICAL QUALITATIVE SOLUTION INDICATING SOME GENERAL TRENDS OF DIVERGING NARROW-CHANNEL FLOW.

error, one can solve the energy equation simultaneously with the equations of motion (Eqn. 7) to asses the exact thermal behaviour of the entrance flow.

\section{Solution procedure}

For a given problem specified by its geometry, the fluid's physical properties and supply and ambient pressure values, the solution procedure is outlined in Fig. 5. An iterative procedure is required to obtain the correct value of the gap entrance pressure $p_{o}$ which ensures ambient conditions at the gap exit. In our case, the method of false position (regula falsi) was used. Due to the nonlinearity of the problem, some care must be taken while choosing the start value $p_{o}^{*}$. Although the velocity profile only reaches the parabolic profile asymptotically, the calculation time can be reduced by switching to an analytical solution (based on the Reynolds equation) when the velocity integral function comes close to $2 / 3$.

For an axisymmetric geometry with a height distribution given by:

$$
H(R)=1+v(1-R)
$$

the Reynolds equation is written as (without hydrodynamic or squeeze contribution):

$$
\frac{1}{R}\left(R H^{3} \frac{d P^{2}}{d R}\right)=0
$$

Integration results in: 


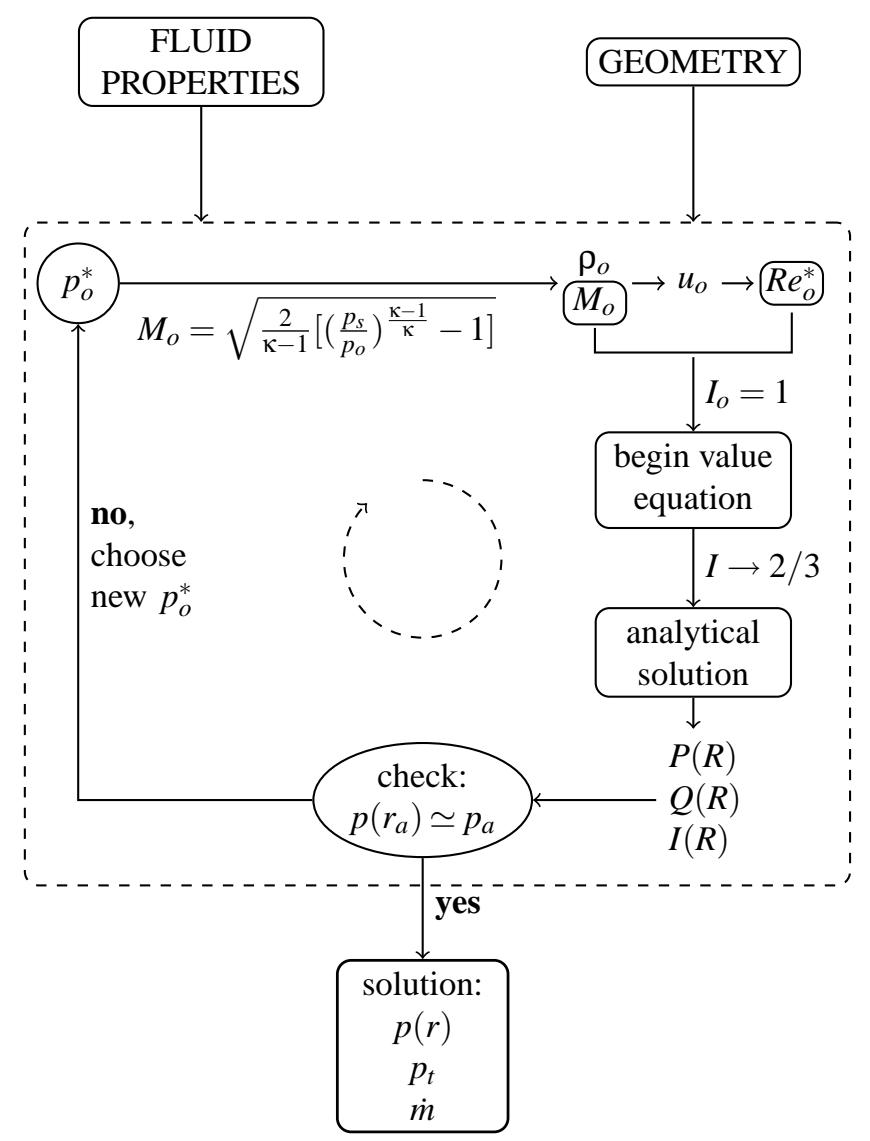

Figure 5. OUTLINE OF THE SOLUTION PROCEDURE

$$
P^{2}=C_{1} B_{v}(R)+C_{2},
$$

where, $(a=1+v)$

$$
B_{\vee}(R)=\frac{1}{a^{3}}\left(\log \left(\frac{R}{H}\right)+\frac{(a+H)^{2}-(1+a)^{2} H^{2}}{2 H^{2}}\right)
$$

Normalisation is performed with respect to ambient $(a)$ or gap entrance conditions $(o)$. Introducing entrance and ambient boundary conditions defines the integration constants $C_{1}$ and $C_{2}$.

\section{APPLICATION OF THE PROPOSED METHOD TO DIF- FERENT FEEDING SYSTEMS}

In this section the proposed solution method is applied to bearing problems with different geometrical configurations and feedings systems. Comparison has been made with experimental data from other authors and with data obtained in our lab. In all following cases the working fluid concerns air. Before making the actual comparison it might be useful to point to some general trends typical to the derived solutions (Fig. 4):

(1) Initially the flow is always accelerating, as in indicated by $Q^{\prime}(1)>0$, independent of the value of the reduced Reynolds number and entrance Mach number;

(2) The pressure curves always feature a characteristic trough, followed by a crest. An increasing value of the reduced Reynolds number $R e_{o}^{*}$ makes this pressure trough more pronounced. This depression has for long been explained by the presence of a "vena contracta" caused by a separation bubble just downstream of the feedhole. This study shows that a pressure depression does not automatically involve the occurrence of separation.

Figure 6 reveals the different feeding systems on which the proposed solution method has been applied. The simplest geometry is the inherent restrictor (a) with a uniform gap height, characterised by the feedhole radius $r_{o}$ and gap height value $h(r)=h_{o}$. The actual restriction occurs at the entrance curtain area given by $2 \pi r_{o} h_{o}$. If a feeding pocket (b) with radius $r_{p}$ and depth $d_{p}$ is provided (mostly for reasons of increased load carrying capacity and/or stiffness, although deep feeding pockets can introduce the phenomenon of "pneumatic hammering" due to the loss of damping), the first restriction takes place at the orifice. A second entrance effect happens when the flow enters the gap, this being comparable in a certain way to an inherently compensated feeding system with $r_{o}=r_{p}$. A last category holds the geometries with a convergent gap height denoted by the conicity angle $\alpha$. These geometries provide superior bearing characteristics as increased load carrying capacity and stiffness.

Figure 7 compares with the experimental data points obtained by Mori et al. [10] for the case of an inherently compensated bearing with a uniform gap height. The agreement is fair, especially regarding the high value of the reduced Reynolds number and entrance Mach number $\left(M_{o}=0.696, R e_{o}^{*}=220.4\right.$ for the lowest of the two curves). The solution indicates the presence of back-flow in the region $1.5 \mathrm{~mm}<r<3 \mathrm{~mm}$.

The next comparison is made against more recent data from Belforte et al. [11]. The test bearing is also of the inherently restricted type, but with a fairly small entrance radius $r_{o}=0.1 \mathrm{~mm}$. Figure 8 shows the derived solution for two different values of the entrance radius. The solution data for $r_{o}=0.1 \mathrm{~mm}$ (value as been mentioned by the authors) yields only qualitative agreement with their experimental data points. Good to excellent agreement is however achieved for an entrance radius value $r_{o}=0.15 \mathrm{~mm}$, even in the region just downstream of the feedhole, as is shown in detail by Fig. 9. Regarding the fact that only this geometry features such a discrepancy, the cause is most likely an inaccuracy in the feedhole fabrication process, either a deviation of the 


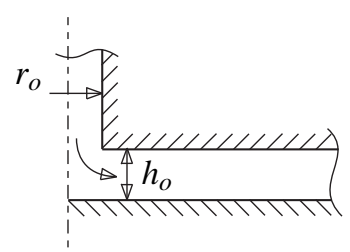

(a)

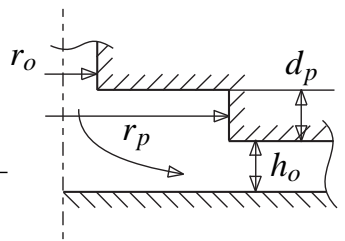

(b)

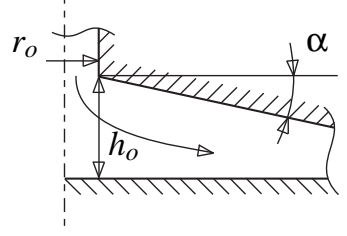

(c)

Figure 6. NOTATION OF THE DIFFERENT FEEDING SYSTEMS: (a) INHERENT RESTRICTOR, (b) ORIFICE WITH FEEDING POCKET, (c) CONVERGENT GAP.

diameter itself or a chamfer at the hole exit, both resulting in an increased entrance curtain area. Configurations combining small entrance radii and thin air gaps are more likely to suffer from the above mentioned effect (as is indicated by all other comparisons having either larger entrance radii or an increased gap height).

Although the solution method was originally intended to be applied to inherently restricted bearing configurations, it can also handle feeding systems which incorporate a pocket/recess. Two distinct approaches are outlined and compared with experimental data. The first approach is to be used on shallow feeding pockets. When entering the bearing gap, the flow passes through two separate entrance curtains, each contributing to the global flow pattern. A first restriction occurs at the curtain given by $2 \pi r_{o}\left(h_{o}+d_{p}\right)$ after which the flow settles down in the feeding recess. From this point, the flow undergoes a second (minor) restriction characterised by $2 \pi r_{p} h_{o}$. Even further downstream, viscous flow sets in up to ambient conditions at gap exit. To model this flow pattern, it is sufficient to introduce a step in the gap height function $h(r)$ at $r=r_{p}$ (a gradual step can prevent numerical problems when integrating the begin value problem). The outlined approach is adopted to the geometry of Fig. 10, demonstrating good agreement with measurements performed by Belforte et al. [11].

For geometries with much deeper pockets, the entrance conditions may be such that severe reverse flow takes place in the recess volume involving the onset of permanent turbulence. At this point the basic assumptions of the solution method are violated

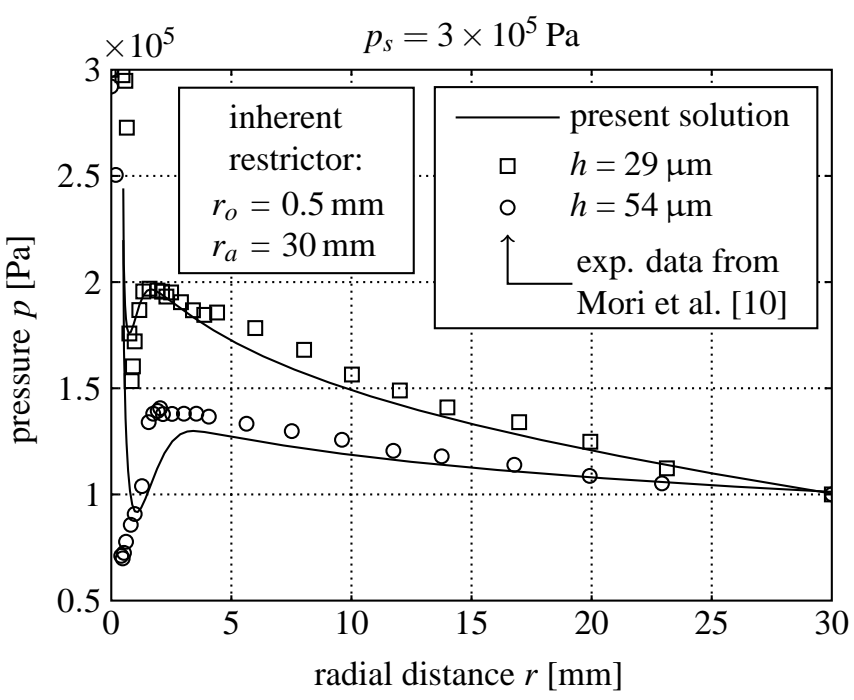

Figure 7. COMPARISON OF PRESSURE PROFILE FOR AN INHERENTLY RESTRICTED AT HIGH MACH EN REYNOLDS NUMBER. $R e_{o}^{*}=57.6, M_{o}=0.568$ FOR $h=29 \mu \mathrm{m} ; R e_{o}^{*}=220.4, M_{o}=$ 0.696 FOR $h=54 \mu \mathrm{m}$.

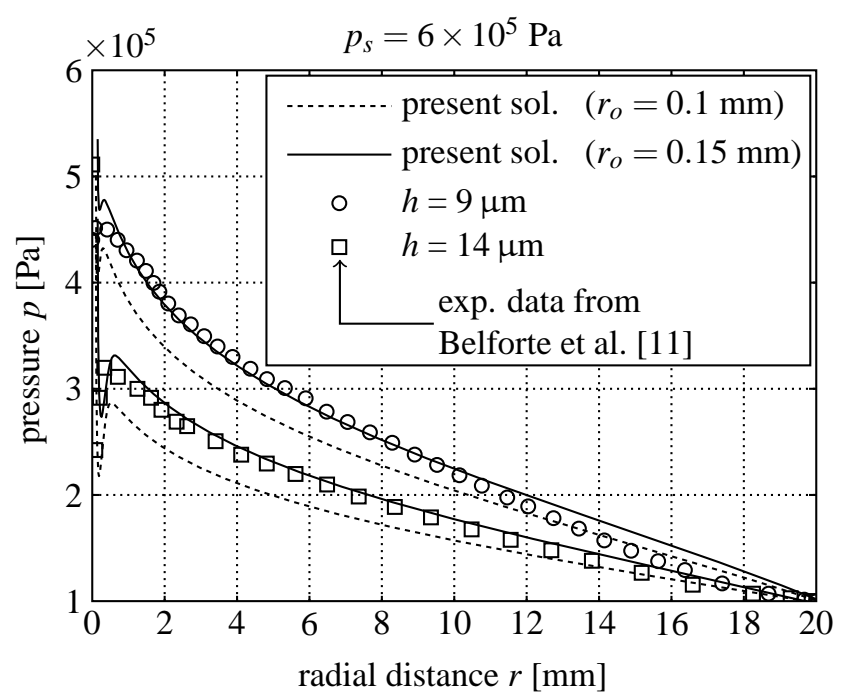

Figure 8. COMPARISON OF PRESSURE PROFILE FOR AN INHERENTLY RESTRICTED BEARING (DETAIL VIEW PROVIDED IN FIGURE 9). $R e_{o}^{*}=27.1, M_{o}=0.407$ FOR $h=9 \mu \mathrm{m} ; R e_{o}^{*}=86.9$, $M_{o}=0.627 \mathrm{FOR} h=14 \mu \mathrm{m}$.

and we have to refer to another solution. For this purpose, a feeding geometry with a deep recess is regarded as a combination of an orifice flow characterised by $r_{o}$ and a certain orifice discharge coefficient $C_{d \text {,orifice }}$ followed by an entrance phenomenon at the curtain denoted by $2 \pi r_{p} h_{o}$. The orifice flow is assumed to loose all its dynamic pressure before leaving the recess volume. An 


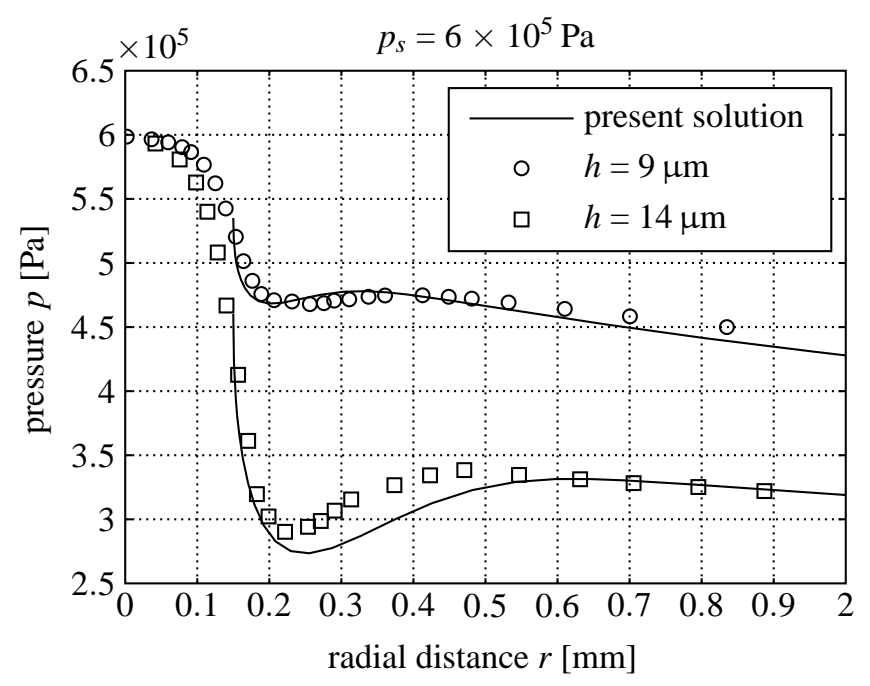

Figure 9. DETAIL VIEW OF FIGURE 8.

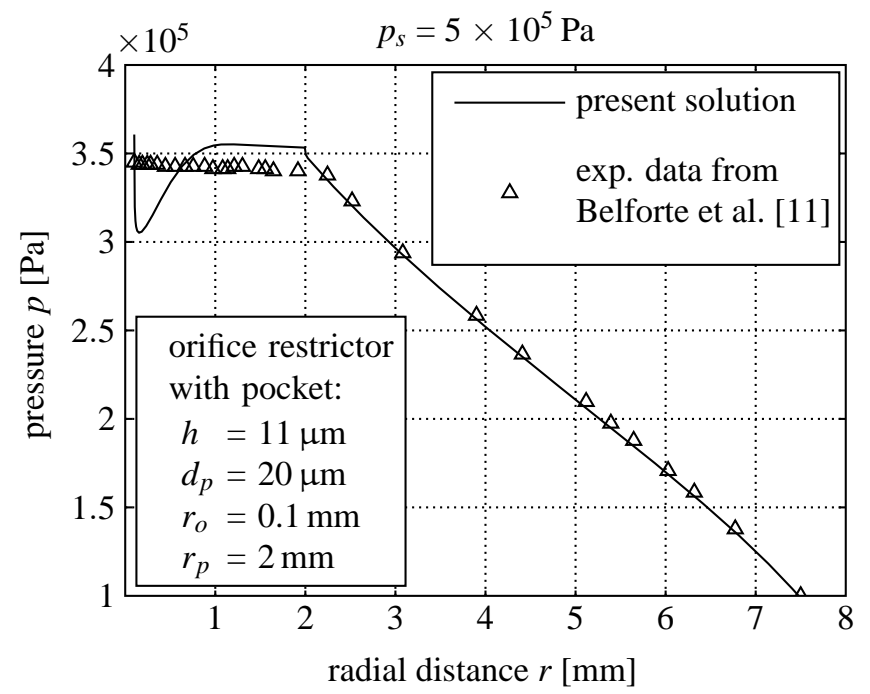

Figure 10. COMPARISON OF PRESSURE PROFILE FOR AN ORIFICE RESTRICTED BEARING WITH A SHALLOW FEEDING POCKET.

iterative solution strategy yields the value of the intermediate recess pressure $p_{r}$ by matching of both flow rates:

$$
\begin{aligned}
& \dot{m}_{1}=f\left(p_{s}, p_{r}, C_{d, \text { orifice }}\right) \\
& \dot{m}_{2}=f\left(p_{r}, p_{o}\right)
\end{aligned}
$$

Figure 11 shows the results obtained in this way. A discharge coefficient $C_{d, \text { orifice }}=0.6$ is assumed for the orifice flow. No information regarding the actual pressure distribution before gap

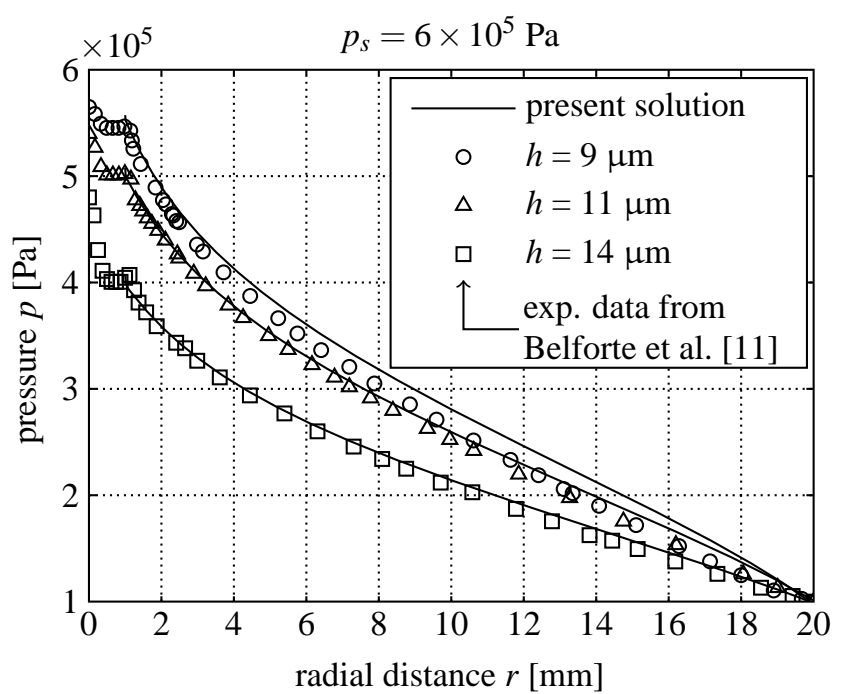

Figure 11. COMPARISON OF PRESSURE PROFILE FOR AN ORIFICE RESTRICTED BEARING WITH A FEEDING POCKET.

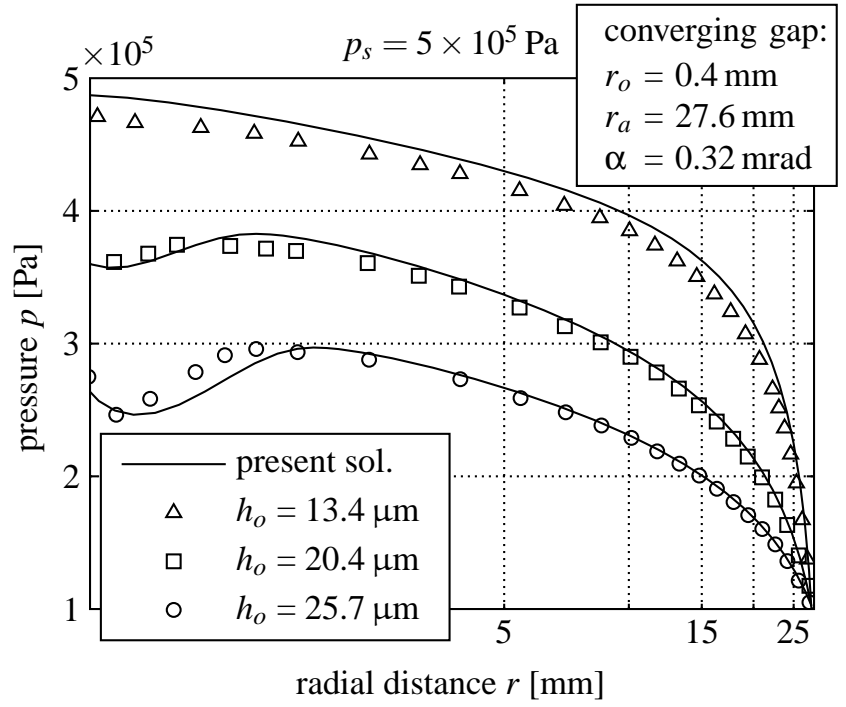

Figure 12. COMPARISON OF PRESSURE PROFILE FOR AN INHERENTLY RESTRICTED BEARING WITH CONVERGENT GAP HEIGHT.

entrance is gained through this method. The comparison should therefore only be made for $r>r_{p}$.

A last validation of the method involves bearing configurations with a converging gap height. Figure 12 displays the comparison with measurements performed in our lab. Besides the good agreement, the superior pressure distribution characteristics of this bearing type are underlined. 


\section{Conclusion and remarks}

The method of separation of variables has been amply demonstrated by means of different bearing configurations and feeding systems. A fair to good agreement is observed over a wide range of geometrical parameters (entrance radius $r_{o}$ and gap height $h_{o}$ ) resulting in varying entrance flow conditions (reduced Reynolds number $R e_{o}^{*}$ and $M_{o}$ ). In a few cases some discrepancy is noticed with experimental data. Besides being caused by inevitable measurement and fabrication errors, some possible explanations are listed:

(1) In the case of small entrance radii and thin air gaps, any surface imperfection (e.g. in the form of burrs, entrance channel chamfering...) affects the entrance curtain geometry to a great extent;

(2) All pressure measurements are recorded by means of a small measuring hole connected through some tubing to a pressure transducer. This measuring hole, albeit small, may change the channel flow to some extent;

(3) The method assumes uniform pressure across the gap, and therefore only predicts an averaged pressure value, while measurements always indicate the wall pressure value. The difference between averaged pressure and wall pressure becomes more pronounced when asymmetry in the flow occurs.

(4) The above stated initial value problem (Eqn. 7) relies on the presence of axisymmetry in the entrance region. For practical bearing configurations, this condition is not always fulfilled. The imposed error is however small since the entrance region of a well designed bearing only constitutes a small part of the total bearing surface.

\section{FORMULATION OF A LUMPED PARAMETER MODEL}

The above outlined and demonstrated method has proven its value for application to various bearing geometries. It would be however more practical to deduce a lumped parameter entrance formulation which combined with the Reynolds equation, results in a fast and easy solution process for a wide variety of bearing problems. The basic idea is to use the theoretical entrance pressure $p_{t}$ (defined by extrapolating the viscous solution back to gap entrance) rather than the pressure at gap entrance $p_{o}$ as an intermediate parameter. The error introduced in this way, is negligible since the entrance flow constitutes only a small fraction of the total bearing surface. In what follows, the definition of an expedient coefficient of discharge will be derived.

The basis of the lumped parameter model is found in the above mentioned nozzle/orifice formula. The difficulty of this formula regards the determination (both empirical or theoretical) of its loss coefficient. To arrive to a new formulation, let us first restate the ideal entrance flow formula (see Eqn. 1 with $A_{o}=$ $\left.2 \pi r_{o} h_{o}\right)$ :

$$
\dot{m}_{o}=A_{o} \sqrt{\frac{2 \kappa}{\kappa-1}} \frac{p_{s}}{\sqrt{\mathfrak{R} T_{s}}} \Phi\left(\frac{p_{o}}{p_{s}}\right)
$$

where we replace $p_{o}$ with $p_{t}$ while introducing the coefficient of discharge $C_{d}$,

$$
\dot{m}_{o}=C_{d} A_{o} \sqrt{\frac{2 \kappa}{\kappa-1}} \frac{p_{s}}{\sqrt{\mathfrak{R} T_{s}}} \Phi\left(\bar{p}_{t}\right)
$$

with,

$$
\bar{p}_{t}=\frac{p_{t}}{p_{s}}
$$

The problem is now to ascertain if this presentation is valid and if so, to determine the form of $C_{d}$. From the previous analysis it is known that:

$$
\begin{aligned}
& \frac{p_{t}}{p_{o}}=f_{1}\left(R e_{o}^{*}, M_{o}, \text { geo }\right) \\
& \frac{p_{o}}{p_{s}}=f_{2}\left(M_{o}\right)
\end{aligned}
$$

"geo" stands for de entrance gap geometrical properties, e.g. uniform gap or convergent gap. Elimination of $p_{o}$ yields:

$$
\bar{p}_{t}=\frac{p_{t}}{p_{s}}=f_{3}\left(\operatorname{Re}_{o}^{*}, M_{o}, \text { geo }\right)
$$

which implies that $C_{d}$ must be of the following form:

$$
C_{d}=C_{d}\left(R e_{o}^{*}, M_{o}, \text { geo }\right)
$$

Since $p_{o}$ has been eliminated, the problem arises to define $R e_{o}^{*}$ and $M_{o}$ (which are directly or indirectly related to $p_{o}$ ). So, a new set of parameters has to be sought that replaces the previous ones. Combining the definitions of the reduced Reynolds number and entrance Mach number (Eqns. 12 and 13) together with the feed flow equation, creates the following relationship:

$$
R e_{o}^{*} \Lambda_{e}=f_{4}\left(M_{o}\right)
$$


where the entrance number $\Lambda_{e}$ depends on the supply pressure, geometry of the problem and on its fluid properties:

$$
\Lambda_{e}=\frac{\mu r_{o}}{p_{s} h_{o}^{2}} \sqrt{\frac{\Re T_{s}}{\kappa}}
$$

Inserting this relation into Eqn. 26 makes clear that both $R e_{o}^{*}$ and $M_{o}$ must be of the form:

$$
\left(\operatorname{Re}_{o}^{*}, M_{o}\right)=f_{5}\left(\Lambda_{e}, \bar{p}_{t}, \text { geo }\right)
$$

Finally, this gives the sought after expression for $C_{d}$ :

$$
C_{d}=f_{6}\left(\Lambda_{e}, \bar{p}_{t}, \text { geo }\right)
$$

The question still remains if this function is single-valued over the entire range of its parameters. The only way to conclude about the uniqueness of $f_{6}$ is to calculate the value of $C_{d}$ for different entrance conditions. This is done by solving the entrance problem for varying values of $\left(R e_{o}^{*}, M_{o}\right)$ as is outlined above. Extrapolating each solution back to gap entrance results in a new combination of $\left(\bar{p}_{t}, C_{d}\right)$. An example of the calculation result for a bearing with a uniform gap height is shown in Fig. 13. Below a certain value of $\bar{p}_{t}$, the onset of turbulence prevents the calculation of a coefficient of discharge. The asymptotic nature of the different curves appears to indicate a constant coefficient of discharge for pressure ratios below this point. The figure also proves the uniqueness of the formulated discharge coefficient for each value of the entrance number $\Lambda_{e}$.

The original begin value problem describing narrow channel flow has now been converted into a more practical lumped parameter formulation by introducing a coefficient of discharge $C_{d}$. Tabulation of this coefficient into a lookup table facilitates the bearing design to a great extent, as will be demonstrated by the following solution procedure (Fig. 14). Given the bearing geometry, working conditions, fluid properties and bearing supply pressure, the total problem of finding the pressure distribution across the bearing gap is split into two subproblems which are coupled through the intermediate pressure value $p_{t}$. Starting with an arbitrary value $p_{t}^{*}$, the first subproblem concerns the solution of the feed flow problem described by Eqn. 22. A second part constitutes of solving the viscous film flow problem in the bearing gap described by the well known Reynolds equation. The value of $p_{t}^{*}$ serves as a boundary condition at feeding sources. Application of a discretisation scheme (finite difference in our case), then yields the static pressure distribution. In case of compressible flow, this requires an iterative solution strategy, e.g.

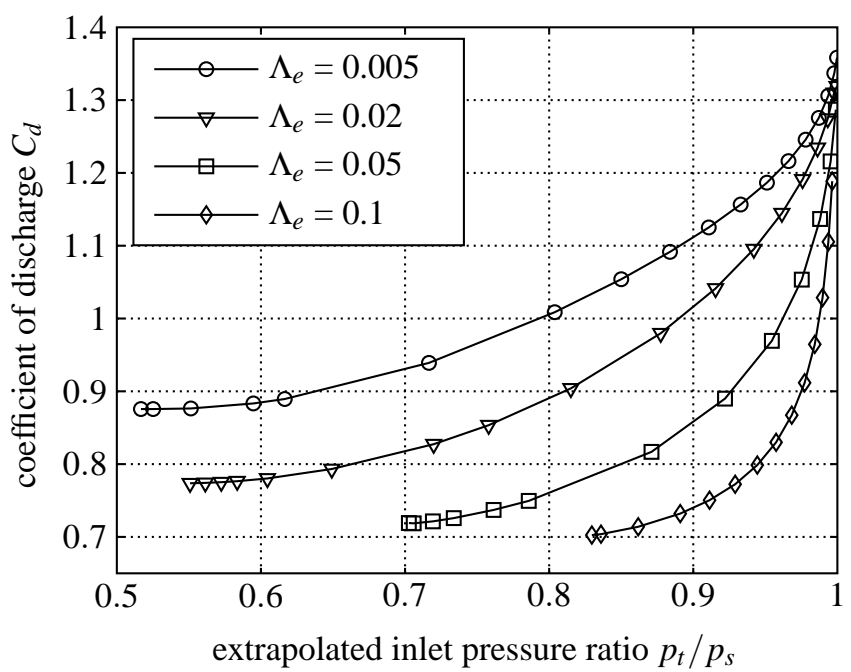

Figure 13. COEFFICIENT OF DISCHARGE AS A FUNCTION OF THE EXTRAPOLATED INLET PRESSURE RATIO FOR A BEARING WITH UNIFORM GAP HEIGHT.

a Newton-Raphson algorithm. Matching of the feed flow rate $\dot{m}_{\text {feed }}$ and the viscous film flow rate $\dot{m}_{\text {film }}$ by means of e.g. the method of false position (regula falsi) leads to the correct value of the intermediate pressure level $p_{t}$ in a few iteration steps. This matching has to be performed for each feeding source, as eccentric operation conditions result in distinct entrance gap heights and therefore different solutions for the theoretical inlet pressure $p_{t}$. Convergence of the matching procedure shows to be somewhat difficult in case of closely spaced feeding sources due to coupling that occurs between the different inlet boundary conditions.

\section{CONCLUSIONS}

The preceding results demonstrate the effectiveness of the proposed solution method in characterising the entrance flow effects encountered in aerostatic bearings over a wide range of entrance parameters. This method takes into account both the Reynolds and Mach numbers at entrance, resulting in an accurate and efficient solution, which can be expressed in terms of the bearing design parameters. It therefore proves its potential as a valid alternative for the various solution methods found in literature, particularly when applying the derived lumped parameter formulation. When compared to empirical solutions, the present solution method relies on an underlying physical model instead of being only the result of experimental data. The method proves to be valid over a wide range of entrance parameters while existing empirical formulas are hardly valid outside the experimental conditions that produced them. Purely numerical solutions, on the other hand, are far less efficient and unhandy for practical bearing design. 


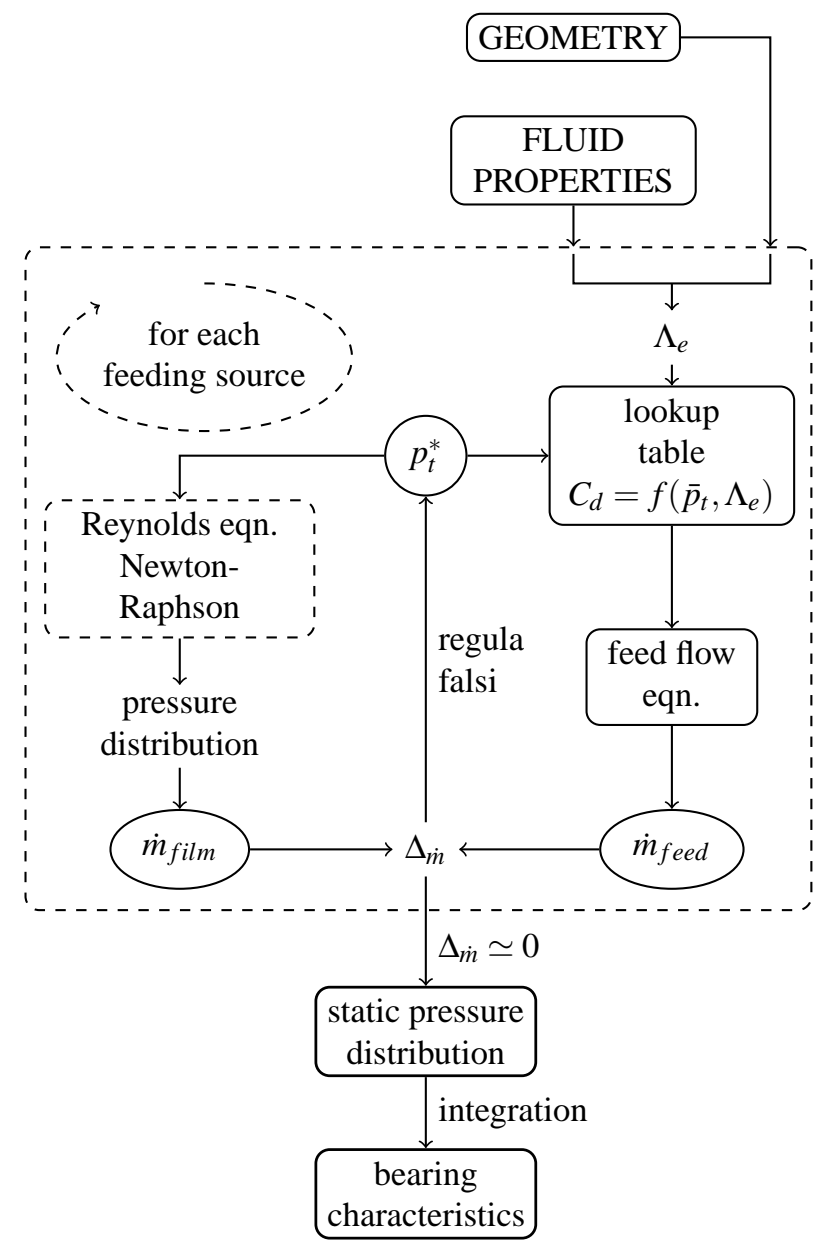

Figure 14. SOLUTION PROCEDURE BY MATCHING OF FEED AND FILM FLOW RATE.

\section{ACKNOWLEDGMENT}

This research is sponsored by the IWT, the Institute for the Promotion of Innovation by Science and Technology - Flanders, Belgium; project SBO 030288 "PowerMEMS". T. Waumans is a research assistant of the Fund for Scientific Research (FWO) Flanders, Belgium.

\section{REFERENCES}

[1] Snoeys, R., and Al-Bender, F., 1978. "Development of Improved Externally Pressurized Gas Bearings". KSME Journal, 1(1), June, pp. 81-88.

[2] Al-Bender, F., and Van Brussel, H., 1992. "Symmetric Radial Laminar Channel Flow With Particular Reference to Aerostatic Bearings". ASME Journal of Tribology, 114(7), July, pp. 630-636.

[3] Vohr, J. H., 1966. An Experimental Study of Flow Phe- nomena in the Feeding Region of an Externally Pressurized Gas Bearing. Report MTI-65TR47, Mechanical Technology Inc., July.

[4] Helene, M., Arghir, M., and Frene, J., 2003. "Numerical Three-Dimensional Pressure Patterns in a Recess of a Turbulent and Compressible Hybrid Journal Bearing". ASME Journal of Tribology, 125(2), April, pp. 301-308.

[5] Braun, M. J., and Dzodzo, M., 1995. "Effects of Hydrostatic Pocket Shape on the Flow Pattern and Pressure Distribution". International Journal of Rotating Machinery, 1(3-4), pp. 225-235.

[6] Renn, J., and Hsiao, C., 2004. "Experimental and CFD study on the mass flow-rate characteristic of gas through orifice-type restrictor in aerostatic bearings". Tribology International, 37(4), April, pp. 309-315.

[7] Al-Bender, F., and Van Brussel, H., 1992. "A Method of "Separation of Variables" for the Solution of Laminar Boundary-Layer Equations of Narrow-Channel Flows". ASME Journal of Tribology, 114(7), July, pp. 623-629.

[8] Tang, I. C., 1968. "Inertia Effects of Air in an Externally Pressurized Gas Bearing”. Acta Mechanica, 5(1), March, pp. 71-82.

[9] Schlichting, H., 1968. Boundary-Layer Theory. , 6th ed., McGraw-Hill, New York.

[10] Mori, H., and Miyamatsu, Y., 1969. "Theoretical Flow-Models for Externally Pressurized Gas Bearings". ASME Journal of Lubrication Technology, 91(1), January, pp. 181-193.

[11] Belforte, G., Raparelli, T., Viktorov, V., and Trivella, A., 2007. "Discharge coefficients of orifice-type restrictor for aerostatic bearings". Tribology International, 40(3), March, pp. 512-521. 